\title{
Trouble in Anatolia
}

Tn this issue of the Canadian Respiratory Journal, Gulmez et al (pages 287-290) review the presentation, management and outcome of malignant mesothelioma in central Anatolia, Turkey. There is nothing particularly new about the disease here; it presents with chest pain, dyspnea and pleural effusion, is diagnosed by biopsy and has a miserable outcome whatever is done for it, just like malignant mesothelioma in Canada. Mesothelioma in Anatolia is unusual in that it is very common, with an approximately equal sex distribution, and frequently occurs in relatively young people. The reasons for this are not a mystery. Anatolia is a

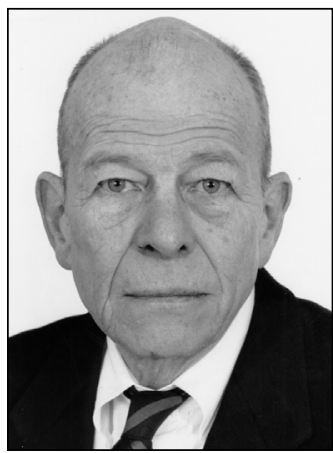

Nick R Anthonisen

Problèmes en Anatolie

volcanic area; there is a lot of asbestos on or near the surface, and it is widely used as construction material, in painting, and as insulation (pages 287-290)! Asbestos related disease is due to environmental, not occupational exposure, and exposure is more intense and occurs at an earlier age than in Canada. Not only that, but some areas of Anatolia are rich in erionite, and people build homes in and on erionite-bearing rocks. Erionite is apparently something like asbestos but worse in terms of carcinogenicity (1), perhaps because its crystals have large surface areas, and areas with erionite have an even higher incidence of mesothelioma.

This is an amazing story, at least to me. When the paper of Gulmez et al (pages 287-290) was submitted, I had just finished reading a book on mesothelioma (1). From the clinical viewpoint, it was not very enlightening; presentation, diagnosis and outcome around the world is similar to that in Canada, or Turkey. It was, however, extremely enlightening in terms of the politics of asbestos exposure in the first world. Asbestos mining and processing had been going on for most of the twentieth century, and asbestosis was well recognized for much of this time. However, with the Second World War, there was an enormous increase in asbestos use. Mesothelioma, as a result of asbestos exposure, was first described in South Africa in the late 1950s, and from the beginning it was clear that heavy asbestos exposure was not necessary. It took 25 years before the carcinogenicity of asbestos was widely recognized by authorities and its health risks became a more important priority than its commercial value. The asbestos market essentially collapsed amidst lawsuits in the mid 1980s and occupational asbestos exposure is now uncommon. In the interim, a well-documented epidemic of mesothelioma occurred in Europe, North America, Australia and even Japan. Since in these areas, mesothelioma occurs 20 to 30 years postexposure, its incidence continues to increase in most of them. Though it appears that it may be declining in North America, it may not do so until 2020 in Australia and the United Kingdom. It is a real horror story and I recommend the book to anyone who is interested in the topic.

I was thus dumbfounded to read that in Central Anatolia asbestos and erionite related mesothelioma is still common apparition chez des personnes relativement jeunes. Les raisons ne sont pas mystérieuses. L'Anatolie est une région volcanique. On y trouve beaucoup d'amiante près de la surface ou en surface, lequel est largement utilisé pour la construction, la peinture et l'isolation (pages 287-290) ! Les maladies causées par l'amiante sont d'origine environnementale et non industrielle, et l'exposition est plus intense et se fait plus tôt dans la vie qu'au Canada. De plus, certaines régions de l'Anatolie sont riches en érionite, et les habitants construisent des maisons dans des rochers contenant de l'érionite ou à l'aide de ces rochers. Apparemment, l'érionite ressemble à l'amiante, mais son effet cancérigène est plus marqué (1), peut-être parce que ses cristaux couvrent de grandes surfaces. Les régions où se trouve l'érionite présentent une incidence encore plus élevée de mésothéliome.

C'est une histoire incroyable, du moins pour moi. Lorsque l'article de Gulmez et coll. (pages 287-290) a été soumis, je venais de finir un livre sur le mésothéliome (1). D’un point de vue clinique, ce n'était pas très instructif. La présentation, le diagnostic et l'issue autour du monde sont semblables à ceux du Canada ou de la Turquie. C'était toutefois très instructif en matière de politiques sur l'exposition à l'amiante dans les pays industrialisés. L'extraction et la prospection de l'amiante se sont poursuivies pendant la plus grande partie du $\mathrm{XX}^{\mathrm{e}}$ siècle, et l'amiantose est connue depuis presque toute cette période. Cependant, avec la Seconde Guerre mondiale, l'usage de l'amiante a beaucoup augmenté. Le mésothéliome, découlant de l'exposition à l'amiante, a été décrit pour la première fois en Afrique du Sud à la fin des années 1950. Depuis le début, il était évident que l'exposition marquée à l'amiante n'était pas nécessaire. Il a fallu 25 ans pour que le caractère cancérigène de l'amiante soit largement admis par les autorités et pour que ses risques pour la santé aient priorité sur valeur commerciale. Le marché de l'amiante s'est à peu près effondré au milieu de poursuites judiciaires dans les années 1980, et l'exposition industrielle à l'amiante est maintenant rare. Entre-temps, une épidémie de mésothéliome bien documentée s'est produite en Europe, en Amérique du Nord, en Australie et même au Japon. Puisque le mésothéliome fait son apparition de 20 à 30 ans après l'exposition, son incidence a continué d'augmenter dans la plupart de ces régions. Il semble peut-être diminuer en 
because people are still using these minerals and are therefore exposed to large quantities of them. Mesothelioma is endemic at high levels there, not epidemic, something that is not widely recognized outside of Turkey; there is no chapter on Turkey's experience with mesothelioma in the 350-page book on the disease (1). One might have thought that something would have been done to limit asbestos exposure in central Anatolia. I suppose that I should not have been surprised. I am, after all, a respiratory specialist who has made a career out of studying smoking related diseases, and in some ways these are similar to mesothelioma in central Anatolia. Like asbestos and erionite, the health effects of smoking occur long after the initial exposure and many people find the inciting agent(s) to be of value. Educational efforts to modify the smoking habits of populations have been a long and difficult process, and I suppose that the same may be true of asbestos use in central Anatolia, though I have no idea whether or not educational efforts have been made. If not, it would seem that they certainly should be. An alternative approach would be to make certain harmful lifestyles illegal, ie, outlaw cigarettes or asbestos use and housing near erionite. We have not done the former, and should probably not criticize the Turkish government for not doing the latter, since they are clearly faced with a more complicated situation and likely have fewer resources to deal with it.

\section{REFERENCE}

1. Robinson BWS, Chahinian AP, eds. Mesothelioma. London: Martin Dunitz Ltd, 2002.
Amérique du Nord, mais il ne le fera peut-être pas avant 2020 en Australie et au Royaume-Uni. C'est une vraie histoire d'horreur. Je recommande l'ouvrage à tous ceux qui sont intéressés par le sujet.

Je suis donc resté stupéfait de lire qu'en Anatolie centrale, le mésothéliome relié à l'amiante et à l'érionite demeure courant parce que les habitants continuent d'utiliser ces minéraux et qu'ils y sont donc très exposés. Le mésothéliome y est endémique à taux élevé, et non épidémique, ce dont on n'a pas beaucoup conscience à l'extérieur de la Turquie. Aucun chapitre ne porte sur l'expérience du mésothéliome en Turquie dans l'ouvrage de 350 pages sur la maladie (1). On aurait cru que des mesures auraient été prises pour limiter l'exposition à l'amiante en Anatolie centrale. J'imagine que je ne devrais pas être surpris. Après tout, je suis un spécialiste des maladies respiratoires qui a fait carrière dans l'étude des maladies reliées au tabagisme et sous certains aspects, elles sont semblables au mésothéliome en Anatolie centrale. À l'instar de l'amiante et de l'érionite, les effets du tabagisme sur la santé surgissent longtemps après la première exposition, et bien des gens trouvent les agents incitatifs intéressants. Les efforts d'éducation pour modifier les habitudes de tabagisme des populations sont un processus long et difficile, et j'imagine que le même constat s'avère quant à l'usage de l'amiante en Anatolie centrale, même si je ne sais pas si des efforts d'éducation ont été entrepris. Dans la négative, ils devraient sûrement l'être. Une autre méthode consisterait à rendre certains modes de vie illégaux, comme d'interdire l'usage de la cigarette ou de l'amiante et la construction à proximité de l'érionite. Nous n'avons pas adopté la première méthode, et ne devrions probablement pas critiquer le gouvernement turc de ne pas adopter la deuxième, puisque, de toute évidence, ce pays affronte une situation plus complexe et possède sûrement moins de ressources pour la régler.

\section{RÉFÉRENCE}

1. Robinson BWS, Chahinian AP, eds. Mesothelioma. London: Martin Dunitz Ltd, 2002. 


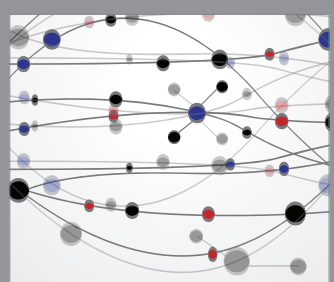

The Scientific World Journal
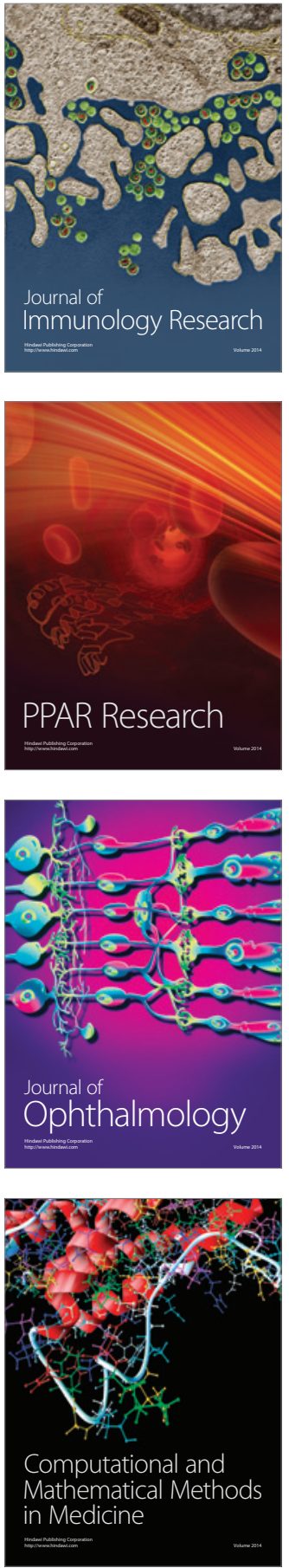

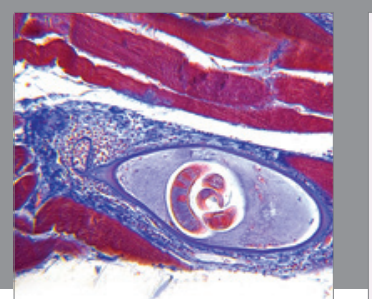

Gastroenterology Research and Practice

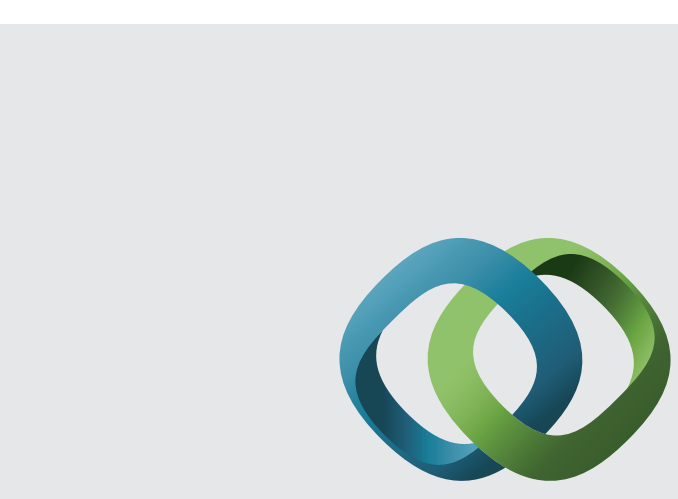

\section{Hindawi}

Submit your manuscripts at

http://www.hindawi.com
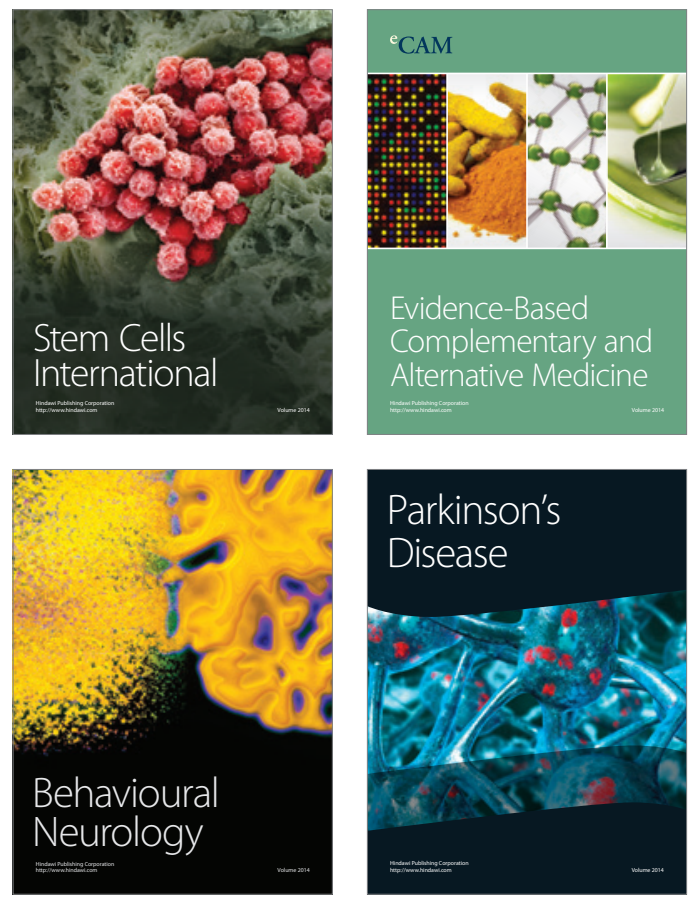
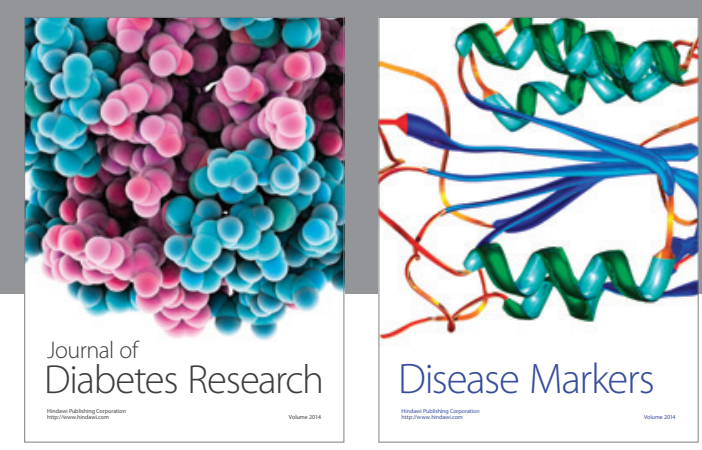

Disease Markers
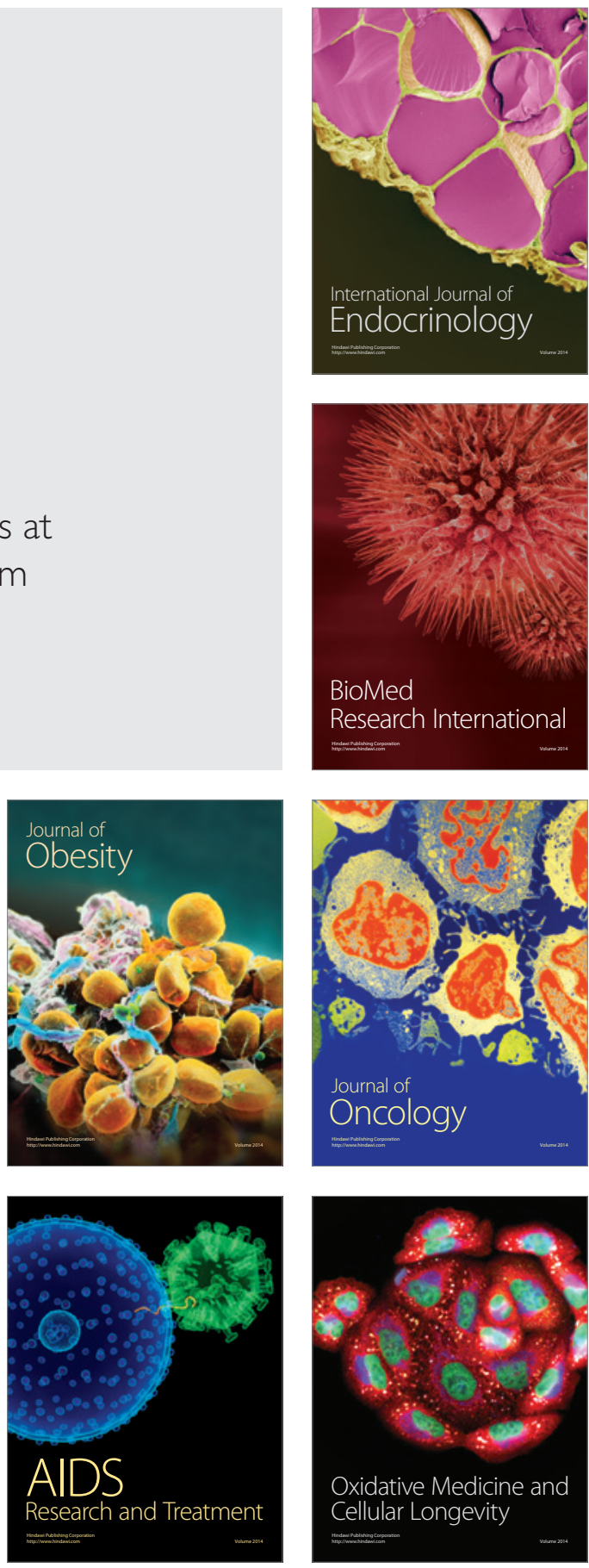